PREPARED FOR THE U.S. DEPARTMENT OF ENERGY, UNDER CONTRACT DE-AC02-76CH03073

PPPL-3923

PPPL-3923

UC-70

Particle-in-cell Simulations with Kinetic Electrons

by

J.L.V. Lewandowski

February 2004

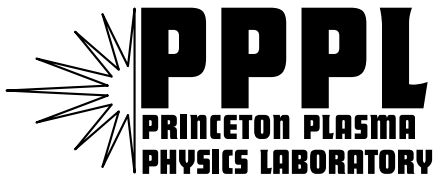

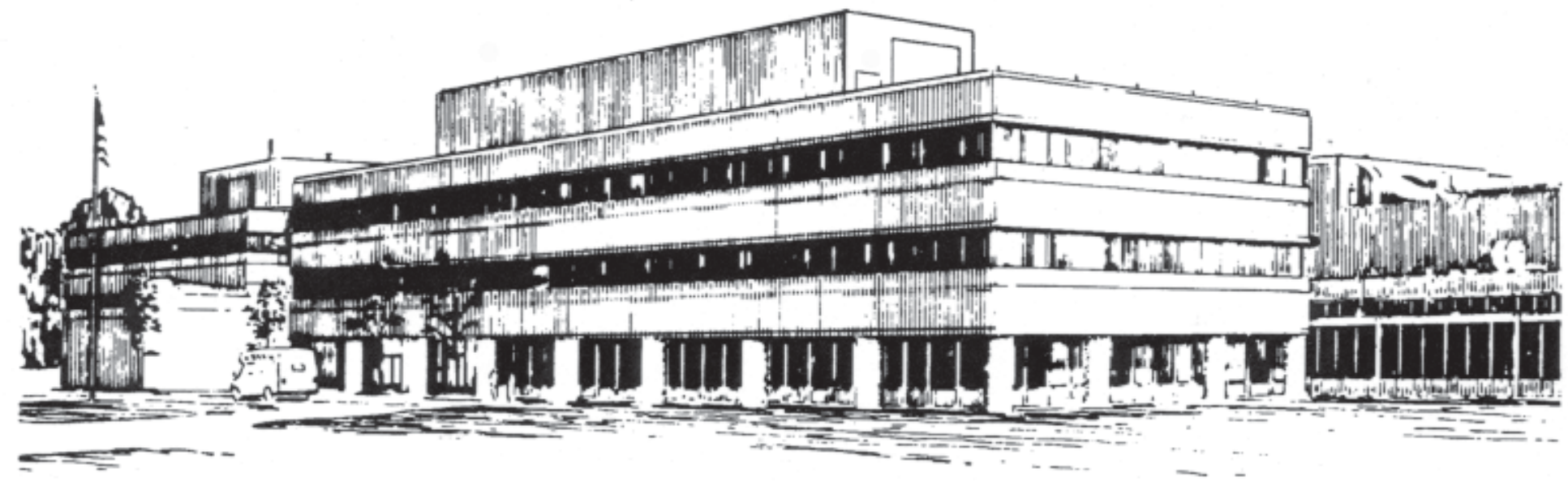

PRINCETON PLASMA PHYSICS LABORATORY PRINCETON UNIVERSITY, PRINCETON, NEW JERSEY 


\section{PPPL Reports Disclaimer}

This report was prepared as an account of work sponsored by an agency of the United States Government. Neither the United States Government nor any agency thereof, nor any of their employees, makes any warranty, express or implied, or assumes any legal liability or responsibility for the accuracy, completeness, or usefulness of any information, apparatus, product, or process disclosed, or represents that its use would not infringe privately owned rights. Reference herein to any specific commercial product, process, or service by trade name, trademark, manufacturer, or otherwise, does not necessarily constitute or imply its endorsement, recommendation, or favoring by the United States Government or any agency thereof. The views and opinions of authors expressed herein do not necessarily state or reflect those of the United States Government or any agency thereof.

\section{Availability}

This report is posted on the U.S. Department of Energy's Princeton Plasma Physics Laboratory Publications and Reports web site in Fiscal Year 2004. The home page for PPPL Reports and Publications is: http://www.pppl.gov/pub_report/

DOE and DOE Contractors can obtain copies of this report from:

U.S. Department of Energy

Office of Scientific and Technical Information

DOE Technical Information Services (DTIS)

P.O. Box 62

Oak Ridge, TN 37831

Telephone: (865) 576-8401

Fax: (865) 576-5728

Email: reports@adonis.osti.gov

This report is available to the general public from:

National Technical Information Service

U.S. Department of Commerce

5285 Port Royal Road

Springfield, VA 22161

Telephone: $1-800-553-6847$ or

(703) $605-6000$

Fax: (703) 321-8547

Internet: http://www.ntis.gov/ordering.htm 


\title{
Particle-in-cell Simulations with Kinetic Electrons
}

\author{
J.L.V. Lewandowski * \\ Princeton Plasma Physics Laboratory \\ Princeton University, P.O. Box 451 \\ Princeton NJ 08543 \\ USA
}

February 3, 2004

\begin{abstract}
A new scheme, based on an exact separation between adiabatic and nonadiabatic electron responses, for particle-in-cell (PIC) simulations of drift-type modes is presented. The (linear and nonlinear) elliptic equations for the scalar fields are solved using a multigrid solver. The new scheme yields linear growth rates in excellent agreement with theory and it is shown to conserve energy well into the nonlinear regime. It is also demonstrated that simulations with few electrons are reliable and accurate, suggesting that large-scale, PIC simulations with electron dynamics in toroidal geometry (e.g. tokamaks and stellarators plasmas) are within reach of present-day massively-parallel supercomputers.
\end{abstract}

Pacs \# : 52.35Kt, 52.30Jb, 52.35Ra

Keywords: Turbulence, particle-in-cell, multigrid, drift-waves

*e-mail: jlewando@pppl.gov 


\section{Introduction}

It is well known that low-frequency, small-scale instabilities (eg. drift waves, Ion Temperature Gradient-driven (ITG) modes) are major contenders for the anomalous, cross-field transport observed in tokamaks [1-3] and stellarators [4]. Although a considerable amount of theoretical and numerical work has been published on the topic of ion-driven microturbulence, the numerical study of electron dynamics for slow, drift-type modes in realistic geometries (e.g. tokamak and stellarator plasmas) has received much less attention. However, the accurate modeling of electron physics represents a most relevant challenge in turbulence simulation research, since electrons are expected to play a key role in the turbulence level and associated transport.

The treatment of electrons in particle-in-cell simulations is made difficult due to the fact that the electrons move $\sim \sqrt{m_{i} / m_{e}} \gg 1$ times faster than the ions. However, for drift-type modes [2, $3]$, the bulk of the electrons respond adiabatically to the waves and it may be advantageous to focus on the nonadiabatic part of the electron response. In this paper, we introduce the splitting scheme, a scheme which naturally segregates the adiabatic part of the electron response from its nonadiabatic part. It shown that, for the same physical and numerical parameters, the splitting scheme yields more accurate linear growth rates and has better energy conservation properties that the conventional $\delta f$ scheme [6]. There is ongoing effort to extend the present scheme to the case of electromagnetic perturbations.

The paper is organized as follows; in section 2, we motivate and describe the splitting scheme; linear and nonlinear field equations are also given. The nonlinear elliptic problems arising in the splitting scheme are solved using a multigrid technique, as discussed in section 3; in addition, the multigrid particle-in-cell algorithm is given in the same section. Section 4 is devoted to the numerical results; a model problem is solved to test the multigrid Poisson solver. Various aspects related to nonlinear saturation through mode coupling, diffusion in velocity space and energy conservation are also discussed. Concluding remarks are presented in section 5 .

\section{Splitting Scheme}

In this section, we introduce the splitting scheme to model electrostatic drift waves in shearless slab geometry. The model equations are the collisionless, electrostatic, gyrokinetic Vlasov equation, in the long-wavelength limit,

$$
\frac{d F_{j}}{d t} \equiv \frac{\partial F_{j}}{\partial t}+\left(v_{\|} \widehat{\mathbf{b}}_{0}+\mathbf{V}_{E}\right) \cdot \nabla F_{j}-\frac{q_{j}}{m_{j}} \widehat{\mathbf{b}}_{0} \cdot \nabla \Phi \frac{\partial F_{j}}{\partial v_{\|}}=0
$$

where $\widehat{\mathbf{b}}_{0}=\mathbf{B}_{0} / B_{0}$ is a unit vector and $\mathbf{V}_{E}=c \widehat{\mathbf{b}}_{0} \times \nabla \Phi / B_{0}$ is the $\mathbf{E} \times \mathbf{B}$ drift velocity. The confining magnetic field is taken to be of the form $\mathbf{B}_{0}=B_{0}(\widehat{\mathbf{z}}+\theta \widehat{\mathbf{y}})$ where $\theta$ is a small parameter. Although the standard $\delta f$ scheme works well for the ion dynamics, an accuracy problem arises when the scheme is used to treat the electron dynamics. As discussed in the Introduction, the problem is, of course, related to the fact that the bulk of the electrons move much faster than the ions; however, for low-frequency modes, only a subset of the electron markers interact with the waves. Therefore, it is natural to separate the electrons into two groups (adiabatic and nonadiabatic) as to reflect their different responses to the low-frequency waves. To do so, we write the distribution $F_{j}$ as

$$
F_{j}=H(\Phi) F_{M j}+h_{j},
$$


where $F_{M j}$ is the Maxwellian distribution for particle species $j$ given by

$$
F_{M j}=\frac{n_{0}}{\sqrt{2 \pi} V_{t h j}} \exp \left(-\frac{1}{2} \frac{v_{\|}^{2}}{V_{t h j}^{2}}\right)
$$

where $n_{0}$ is the background density and $V_{t h j}=\sqrt{T_{j} / m_{j}}$ is the thermal velocity for species $j$. In Eq. $(2), H(\Phi)$ is a yet undetermined function of the electrostatic potential. The so-called nonadiabatic part of the distribution function, $h_{j}$, is governed by

$$
\frac{d h_{j}}{d t}=-H \underbrace{\mathbf{V}_{E} \cdot \boldsymbol{\nabla} F_{M j}}_{\mathbf{1}}-v_{\|} \nabla_{\|} \Phi \underbrace{\left(H^{\prime}+\frac{q_{j}}{T_{j}} H\right)}_{\mathbf{2}} F_{M j}-\underbrace{H^{\prime} F_{M j} \frac{\partial \Phi}{\partial t}}_{\mathbf{3}},
$$

where a prime denotes a derivative with respect to $\Phi$ and the relation $\mathbf{V}_{E} \cdot \boldsymbol{\nabla} \Phi=0$ has been used. Term 1 represents the drive from the free energy whereas term $\mathbf{2}$ accounts for the parallel free streaming and the parallel force. Demanding that term $\mathbf{2}$ vanishes, we obtain

$$
F_{j}=\exp \left(-\frac{q_{j}}{T_{j}} \Phi\right) F_{M j}+h_{j}
$$

As it will be shown in the next sections, it is numerically more efficient to consider the nonadiabatic response (normalized to $F$ ) instead of the full distribution itself; the quantity of interest, for particle species $j$, is $\bar{W}_{j}(x, v, t) \equiv h_{j}(x, v, t) / F_{j}(x, v, t)$. In the spirit of particle methods, a set of markers is introduced in order to sample the phase space; each marker $k$ has an associated weight $\bar{w}_{j k}$, and $\bar{W}_{j}(x, v, t)$ can be approximated as [5]

$$
\bar{W}_{j}(x, v, t)=\sum_{k=1}^{k=N_{j}} \bar{w}_{j k}(t) \delta\left(x-x_{j k}(t)\right) \delta\left(v-v_{j k}(t)\right)
$$

where $N_{j}$ is the number of markers of species $j$. The main computational effort is now reduced to the computation of the trajectories $\left\{x_{j k}(t), v_{j k}(t), \bar{w}_{j k}(t) ; \forall j, \forall k\right\}$. The equation governing the weight associated with the nonadiabatic part of the distribution function can be easily derived using Eqs. $(1,5)$

$$
\frac{d \bar{w}_{j k}}{d t}=\left(1-\bar{w}_{j k}\right)\left(\mathbf{V}_{E} \cdot \boldsymbol{\kappa}_{j}+\frac{q_{j}}{T_{j}} \varphi\right)
$$

where $\varphi \equiv \partial \Phi / \partial t$ and $d / d t$ denotes the Lagrangian derivative (that is the derivative along the marker trajectory). Here, $\boldsymbol{\kappa}_{j}=\boldsymbol{\kappa}\left[1-\frac{\eta_{j}}{2}\left(1-\bar{v}_{\|}^{2}\right)\right], \bar{v}_{\|}=v_{\|} / V_{t h j}, \boldsymbol{\kappa}=-\nabla n_{0} / n_{0}, L_{n}=$ $-\left(d \ln n_{0} / d x\right)^{-1}, L_{T_{j}}=-\left(d \ln T_{j} / d x\right)^{-1}$ and $\eta_{j}=L_{n} / L_{T_{j}}$. By construction, the contribution due to the free streaming markers has been removed from the weight equation (See Appendix for a comparison with the standard $\delta f$ scheme [6]). Representation (5) implies the computation of an additional scalar field, $\varphi$, as it is apparent in the weight equation (6). Taking the time derivative of the gyrokinetic Poisson (in the long-wavelength limit)

$$
\frac{e^{2}}{T_{e}} n_{0} \rho_{s}^{2} \nabla_{\perp}^{2} \Phi=-\rho \equiv e \int_{-\infty}^{+\infty}\left(F_{e}-F_{i}\right) d v_{\|}
$$


we obtain $e^{2} T_{e}^{-1} n_{0} \rho_{s}^{2} \nabla_{\perp}^{2} \varphi=-\partial \rho / \partial t$. In turn, the quantity $\partial \rho / \partial t$ can be obtained by taking the time derivative of the zeroth-order velocity moment of the Vlasov equation, Eq.(1), with the result of

$$
\frac{\partial \rho}{\partial t}=-\mathbf{V}_{E} \cdot \nabla \rho-\nabla_{\|} J_{\|}
$$

where $J_{\|}$is the parallel current density. In gyrokinetic units $\left(\omega_{c i} t \mapsto t ; v_{\|} / c_{s} \mapsto v_{\|} ; \rho_{s} \boldsymbol{\kappa} \mapsto \boldsymbol{\kappa}\right.$; $\rho_{s} \boldsymbol{\nabla} \mapsto \nabla ; e \Phi / T_{e} \mapsto \Phi$, together with the definitions of $c_{s}=\sqrt{T_{e} / m_{i}}, \omega_{c i}=e B_{0} /\left(m_{i} c\right)$ and $\left.\rho_{s}=c_{s} / \omega_{c i}\right)$, the elliptic equation governing $\varphi$ is given by

$$
\nabla_{\perp}^{2} \varphi=\mathbf{V}_{E} \cdot \nabla \rho+\nabla_{\|} J_{\|}
$$

whereas the gyrokinetic Poisson equation becomes

$$
\nabla_{\perp}^{2} \Phi-\left(1+\frac{1}{\tau}\right) \Phi=\int_{-\infty}^{+\infty}\left(h_{e}-h_{i}\right) d v_{\|}+Q(\Phi)
$$

where $Q(\Phi) \equiv \exp (\Phi)-\exp (-\Phi / \tau)-(1+1 / \tau) \Phi$ and $\tau=T_{i} / T_{e}$. Eq. $(9)$ has been obtained by using representation (5) in Eq.(7). In particular, the electron and ion distribution functions are (in gyrokinetic units)

$$
\begin{aligned}
& F_{i}=\exp \left(-\frac{\Phi}{\tau}\right) F_{M i}+h_{i}, \\
& F_{e}=\exp (\Phi) F_{M e}+h_{e} .
\end{aligned}
$$

Substituting these expressions in Eq.(7) and integrating over velocity space, one gets

$$
\nabla_{\perp}^{2} \Phi=\exp (\Phi)-\exp (-\Phi / \tau)+\int_{-\infty}^{+\infty}\left(h_{e}-h_{i}\right) d v_{\|}
$$

Subtracting $(1+1 / \tau) \Phi$ from both sides of the above equation one obtains Eq.(9). Note that the Maxwellian part of $F_{j}$ does not contribute to the current density since the odd moments of $F_{M j}$ vanish

$$
\int_{-\infty}^{+\infty} F_{M j} v_{\|}^{2 p+1} d v_{\|} \equiv 0
$$

for $p=0,1,2, \cdots$. In summary, the equations describing the splitting scheme are Eq.(6) for the nonadiabatic weight, the elliptic equations $(9,8)$ for $\Phi$ and $\varphi=\partial \Phi / \partial t$, respectively, and the equations of motion (in gyrokinetic units)

$$
\begin{gathered}
\frac{d \mathbf{r}}{d t}=v_{\|} \widehat{\mathbf{b}}_{0}+\widehat{\mathbf{b}}_{0} \times \nabla \Phi \\
\frac{d v_{\|}}{d t}=-Z_{j} \frac{m_{i}}{m_{j}} \nabla_{\|} \Phi
\end{gathered}
$$

Note that the splitting scheme has been used for both ions and electrons. However, the splitting scheme is most useful for the electron population for reasons mentioned in the Introduction. In addition, note that the scheme presented here requires the solution of two field equations for $\Phi$ 
and $\varphi$. The justification for this approach is that a finite difference approximation of $\varphi=\partial \Phi / \partial t$ in the right-hand side of Eq.(6) has been found to be numerically unstable.

In order to benchmark our multigrid particle-in-cell we compare the measured linear growth rate (computed from the time evolution of the electrostatic potential) with the theoretical linear growth rate. In order to determine the linear dispersion relation, one linearizes Eq.(4) and substitutes the resulting expressions for $h_{e}$ and $h_{i}$ in the definitions of $\rho$ and $J_{\|}$. Upon linearization of the field equations, Eqs. $(8,9)$, one obtains the linear dispersion relation of

$$
\left(1+\frac{1}{\tau}+b\right) \omega=-\omega\left[\zeta_{e} Z\left(\zeta_{e}\right)+\frac{1}{\tau} \zeta_{i} Z\left(\zeta_{i}\right)\right]+\omega_{\star}\left[\zeta_{e} R\left(\zeta_{e}\right)-\zeta_{i} R\left(\zeta_{i}\right)\right]
$$

where $R\left(\zeta_{j}\right) \equiv\left(1-\eta_{j} / 2\right) Z\left(\zeta_{j}\right)+\eta_{j} \zeta_{j}\left[1+\zeta_{j} Z\left(\zeta_{j}\right)\right] / 2, \zeta_{j} \equiv \omega /\left(\sqrt{2} k_{\|} V_{t h j}\right), b=k_{y}{ }^{2} \rho_{\mathrm{s}}{ }^{2}$ and $Z(\zeta)$ is the plasma dispersion function of Fried and Conte [7] with argument $\zeta$. The dispersion relation (12) is exactly that obtained by Horton using a standard $\delta f$-type approach [3].

\section{$3 \quad$ Numerical Method}

This section is divided in two parts: the first part deals with the numerical algorithm, whereas the second part describes the multigrid solver used to solve the nonlinear elliptic governing the electrostatic potential $\Phi$ [Eq.(9)].

\subsection{Numerical Algorithm}

The numerical algorithm can be divided in three fundamental steps. In the first step, one computes the zeroth and first order velocity moments of the perturbed parts of the ion and electron distributions; these quantities are used to determine the charge density, $\rho$, and the parallel current, $J_{\|}$, that act as source terms in the field equations for $\Phi$ and $\varphi$ [see Eq.(9) and Eq.(8), respectively]. The second step involves the solution of the linear and nonlinear elliptic equations governing $\Phi$ and $\varphi$ [Eq.(9,8)]. The third step is the 'particle push' (strictly speaking, the marker push): the equations of motions [Eqs.(10,11)] and the marker weight equation [Eq.(6)] are advanced by one time step. Note that the marker weights $\bar{w}_{j k}$ carry the information about the perturbed distribution function (recall the definition of the marker weight: $\bar{w}_{j k} \equiv h_{j} / F_{j}$ ). The algorithm can be summarized as follows:

\section{Multigrid Particle-in-cell Algorithm}

- 1. Grid Deposition: Compute the charge density and the parallel current density on the grid:

$$
\begin{aligned}
\rho & \propto \int_{-\infty}^{+\infty}\left(h_{i}-h_{e}\right) d v_{\|}, \\
J_{\|} & \propto \int_{-\infty}^{+\infty} v_{\|}\left(h_{i}-h_{e}\right) d v_{\|}
\end{aligned}
$$

- 2. Field Solve: Solve the elliptic equations for $\Phi$ and $\varphi$ using a multigrid solver;

- 3. Particle Push: Advance the position in configuration space, the velocity and the weight for each marker;

- 4. Goto 1 (repeat $N$ times). 
Here, $N$ denotes the total number of time steps. The time advance (step 3 ) is carried out using a second-order Runge-Kutta algorithm. For example, consider the evolution equation for the marker weight, Eq.(6), which can be written as

$$
\frac{d \bar{w}_{j k}}{d t}=S_{j k}
$$

where

$$
S_{j k} \equiv\left(1-\bar{w}_{j k}\right)\left(\mathbf{V}_{E} \cdot \boldsymbol{\kappa}_{j}+\frac{q_{j}}{T_{j}} \varphi\right) .
$$

Note that $\Phi$ (required in $\left.\mathbf{V}_{E} \propto \nabla \Phi\right)$ and $\varphi$ in the above equation are evaluated at the particle position by using linear interpolation from the grid quantities. If $n$ denotes the current time step, the marker weight at the next time step is obtained from

- $\bar{w}_{j k}^{\left(n+\frac{1}{2}\right)}=\bar{w}_{j k}^{(n)}+\frac{\Delta t}{2} S_{j k}^{(n)}$,

- $\bar{w}_{j k}^{(n+1)}=\bar{w}_{j k}^{(n)}+\Delta t S_{j k}^{\left(n+\frac{1}{2}\right)}$.

The computation of the intermediate quantity $S_{j k}^{\left(n+\frac{1}{2}\right)}$ is carried out through steps $\mathbf{1}$ (grid deposition) and $\mathbf{2}$ (field solve) in the multigrid PIC algorithm. The equations of motions [Eqs.(10,11)] are advanced in time using the same method. The equation of motions and the marker weight equation are advanced with a time step $\Delta t$ such that

$$
\omega \Delta t \ll 1
$$

where $\omega$ is the typical frequency of the mode of interest. Drift waves are low-frequency waves with $\omega \ll \omega_{c i}$; in practice a normalized time step, $\Delta \bar{t} \equiv \omega_{c i} \Delta t$, of about one or less is sufficient to resolve all the modes of interest. Better energy conservation can of course be achieved by using small time steps.

At the beginning of the simulation, the markers are distributed randomly in configuration space; their velocities are determined from a Maxwellian distribution [Eq.(3)]. A $t=0$, the marker weights, which are related to the amplitude of the perturbed distribution function, are given by

$$
\bar{w}_{j k}=\left(\xi_{j}-\frac{1}{2}\right) \epsilon,
$$

where $\xi_{j}$ is a random number uniformly distributed between 0 and 1 , and $\epsilon$ is a small parameter (typically $\epsilon=10^{-3}$ ).

\subsection{Multigrid Poisson Solver}

One consequence of the splitting scheme is that the original (linear) gyrokinetic Poisson equation, Eq.(7), is transformed into a nonlinear elliptic problem, Eq.(9).

It can be argued that the nonlinear term, given by $Q(\Phi)$, in the new form of the gyrokinetic Poisson is small [i.e. $\mathcal{O}\left(|\Phi|^{2}\right)$ ] and that it can be neglected. However, higher-order terms such $\Phi^{2}, \Phi^{3}, \ldots$, although small in amplitude, do generate shorter wavelength modes. Short wavelength modes must be retained when considering the energy conservation associated with any new algorithm. Although the original Poisson equation, Eq.(7), can be solved using Fast Fourier 
Transform (FFT) techniques, this is not true of Eq.(9) which contains an infinite number of nonlinear terms. Therefore, we must resort to grid-based techniques such as relaxation methods. In this paper, we use a multigrid solver to solve the elliptic equations governing $\Phi$ and $\varphi$.

The fundamental idea behind the multigrid method $[8,9]$ is to solve modified problems using different scales (grids) in order to suppress error components of different scales. Basic iterative methods (e.g. Jacobi method, Gauss-Seidel method,...) are very efficient at damping short wavelength error components but converge poorly for the long wavelength error components. For clarity, we consider the following model problem of a configuration with period $L$

$$
L F \equiv \frac{d^{2} F}{d \theta^{2}}=S(\theta)
$$

where $\theta \equiv 2 \pi x / L$ is an angle-like coordinate with period $2 \pi$ and $S(\theta)$ is a known source term. The uniform computational grid nodes has $N_{\theta}$ nodes. Problem (13) can be defined on a set of overlapping grids (or levels) as

$$
L^{(p)} F^{(p)}=S^{(p)},
$$

where $p=0,1, \cdots, Q$, and $Q$ is the total number of levels compatible with the original number of grid points $N_{\theta}$; the grid spacing on the finest grid $(p=0)$ is $\Delta \theta=2 \pi / N_{\theta}$, whereas the grid spacing on coarser grids is $\delta \theta=2^{p} \Delta \theta$ (for $p>0$ ). If $f$ denotes the exact solution of Eq.(13), that is $f=L^{-1} S$, and if $F$ denotes an approximation to the exact solution, then the algebraic error, $e \equiv f-F$, and the residual $r \equiv S-L F$ and related through the residual equation

$$
L e=r \quad\left(\text { or } L^{(p)} e^{(p)}=r^{(p)} .\right)
$$

If $A^{(p)}$ denotes an approximation to $L^{(p)^{-1}}$ then $F^{(p)}=A^{(p)} S^{(p)}$; in general, the approximate solution $F^{(p)}$ will depend on the initial guess $F_{0}^{(p)}$. Thus

$$
F^{(p)}=A^{(p)}\left(S^{(p)} ; F_{0}^{(p)} ; \nu_{p}\right)
$$

where $\nu_{p}$ is the number of relaxation sweeps on level $p$ and $F_{0}^{(p)}$ is the initial guess. We define the intergrid transfer operators from coarse grid to fine grid , $I(p+1 \mapsto p)$ (prolongation operator), and from fine grid to coarse grid, $I(p \mapsto p+1)$ (restriction operator). In this paper we focus our attention on the so-called (multigrid) $V$ cycle algorithm; in the $V$ cycle, one proceeds from the finest grid $(p=0)$ to the coarsest grid $(p=Q)$, and then back to the finest grid. The $V$ cycle can be cast in the following algorithmic form of

MultiGrid V cycle

$$
\begin{aligned}
F^{(p)} & =A^{(p)}\left(S^{(p)} ; F_{0}^{(p)} ; \nu_{p}\right) \\
r^{(p)} & =S^{(p)}-L^{(p)} F^{(p)} ; p=0,1, \cdots, Q-1 \\
S^{(p+1)} & =I(p \mapsto p+1) r^{(p)} \\
\ldots \ldots & \cdot \ldots \ldots \ldots \ldots \ldots \ldots \ldots \ldots \ldots \ldots \\
F_{0}^{(p)} & \Longleftarrow F^{(p)}+I(p+1 \mapsto p) F^{(p+1)} \\
F^{(p)} & =A^{(p)}\left(S^{(p)} ; F_{0}^{(p)} ; \nu_{p}\right)
\end{aligned}
$$

The dotted line indicates the bottom of the $V$ cycle. For a nonlinear problem (when the righthand side of Eq.(13) depends nonlinearly on $F$ or its derivatives), one can simply use Newton's method to linearize the right-hand side; typically a few Newton iterations are required. 


\section{Numerical Results}

In this section, a model problem is used to illustrate the efficiency of the multigrid Poisson solver. Linear growth rate measurements, which are used as a tool to benchmark the code, are then presented. The comparison with theory is also discussed for a nonlinear case (nonlinear saturation due to the parallel nonlinearity). The advantages of the splitting scheme (noise free simulations, small number of markers per cell and good conservation properties) are also illustrated with specific numerical examples. The characterization of the drift wave turbulence in velocity space is also discussed.

In order to illustrate the usefulness and the efficiency of the multigrid Poisson solver, consider Eq.(13) with a source term of the form

$$
S(\theta)=-\sum_{q=1}^{M} q^{2}\left[\widehat{\alpha}_{q} \cos (q \theta)+\widehat{\beta}_{q} \sin (q \theta)\right],
$$

which corresponds to the exact solution

$$
f(\theta)=\sum_{q=1}^{M} \widehat{\alpha}_{q} \cos (q \theta)+\sum_{q=1}^{M} \widehat{\beta}_{q} \sin (q \theta),
$$

and $M$ is the total number of modes; such exact solution allows us to 'tune' specific modes and study the performance of the multigrid solver for short-, intermediate- and long-wavelength modes. For the present case, we have chosen $M=12$ modes with amplitudes $\widehat{\alpha}_{q}=\widehat{\beta}_{q}=1$, for $q=1,2, \cdots, M$. The initial profile (on the finest grid) is chosen as

$$
F_{0}(\theta)=\pi^{2} \sin (\theta / 2)
$$

Figure 1 shows the $L^{2}$ norm of the residual as a function of the number of the $V$ cycles for a grid with $N_{\theta}=256$ nodes. The number of relaxations per level is kept fixed at $\nu=4$; the basic solver on each level is the damped Jacobi method with damping parameter $\omega=0.6$. We note the sharp decrease in the $L^{2}$ norm of the residual after a single $\mathrm{V}$ cycle. The discretization error is reached after $9 \mathrm{~V}$ cycles. As a comparison, it takes approximately 7000 iterations to solve the same problem with the same accuracy when using a single-grid algorithm! This difference in computational efficiency between single-grid algorithms and multigrid algorithms becomes more acute when the number of unknowns increases. The multigrid solver described in the previous section is now used to compare the standard $\delta f$ scheme and the splitting scheme for the case of electrostatic drift waves.

We first discuss some linear simulation results. Figure 2 shows the linear growth rate obtained using the $\delta f$ scheme (triangles) and the splitting scheme (squares). The plain line is the numerical solution (based on Muller's algorithm [10] in the complex $\omega_{r}-\gamma$ plane) of the dispersion relation (12); the parameters are: $N_{i}=6765$ (number of ion markers); $N_{e}=6765$; system length $L=8$ for a grid with 64 nodes; the time step step is $\Delta t=1.0$. The magnetic field tilt is $\theta=0.01$, and the electron and ion temperature-gradient parameters are $\eta_{e}=\eta_{i}=0$. The driving parameter, $\kappa=\rho_{s} / L_{n}$, has been varied between 0.05 and 0.13 .

For the splitting scheme, the gyrokinetic Poisson equation, Eq.(9), has been solved using the multigrid solver described above. Newton's method is used to treat the term $Q(\Phi)$ in Eq. $(9)$; typically 2 to 3 Newton iterations are sufficient for convergence. For all the simulations presented in this paper, the multigrid relaxations have been carried out with a set of $6 V$ cycles, with $\nu=4$ relaxations on each grid level. 
In Figure 2, the splitting scheme yields a linear growth in excellent agreement with the theory; the standard $\delta f$ scheme, however, is quite inaccurate even when the drive is strong. If the number of markers is increased, both the $\delta f$ scheme and the splitting scheme become more accurate since the sampling of phase space is more detailed. However, for a given number of markers, the performance of the $\delta f$ scheme always lags behind the performance of the splitting scheme; in fact, 'imperfect' splitting schemes which involve the parallel velocity in the marker weight evolution must be less performant that the 'exact' splitting scheme described in this paper.

Figure 3 shows the real and imaginary parts of the electrostatic potential for a nonlinear run. The parameters are $N_{e}=N_{i}=10946$ and $\kappa=1.15$. Other parameters are the same as in Figure 2. We note that this clean result has been obtained for a relatively small number of markers, and it is in good agreement with the saturation level based on a three-wave mode coupling theory [11] (see also Figure 4).

The nonlinear saturation of the most unstable modes $(n \pm 1)$ is due to the parallel nonlinearity; in this simplified one-dimensional model, the saturation due to the $\mathbf{E} \times \mathbf{B}$ advection is absent. In a more realistic situation (e.g. toroidal plasmas), both mechanisms can contribute to saturate the drift waves. Based on a perturbative, three-wave coupling theory (two fastest growing modes, $\delta f_{ \pm 1}$, and the nonlinear $n=0$ response, $\delta f_{0}$, due to mode coupling), Parker and Lee have estimated that the saturation level of $\Phi(n=1)$ to be [11]

$$
|\Phi|_{\text {sat }}=5.48\left(\frac{\gamma_{\ell}}{k_{\|} V_{\text {the }}}\right)^{2},
$$

where $\gamma_{\ell}$ is the linear growth rate and $k_{\|}=\theta k_{\perp}$ in this one-dimensional model. Figure 4 shows the measured saturation level for the $n=1$ mode as a function of the drive, $\kappa$, for a simulation with $N_{i}=N_{e}=6765$ markers. Since the linear growth rate varies linearly with the density gradient parameter, we expect $|\Phi|_{\text {sat }} \propto \kappa^{2}$. The plain line in Figure 4 shows the theoretical value [Eq.(21)] whereas the squares and the triangles denote the simulation results for $N_{e}=N_{i}=6765$ and $N_{e}=N_{i}=4181$, respectively. Taking into account that these simulations have been carried out with a small number of markers, the agreement with theory is quite satisfactory. Figure 4 shows that the agreement with theory improves considerably when the number of markers is increased from $N_{e}=N_{i}=4181$ (triangles) to $N_{i}=N_{e}=6765$ (squares).

In order to further assess the advantages of the splitting scheme, we have performed a scan in mode number (i.e. a variation of $k_{\perp} \rho_{s}$ ) and studied the linear growth rate for various number of electron markers, $N_{e}$; this is illustrated in Figure 5. The parameters are for a grid with $N=512$ nodes; the number of ion markers is kept fixed at $N_{i}=10946$. The squares, diamonds and triangles in Figure 5 represent the linear growth rates for the cases of $N_{e}=144, N_{e}=233$ and $N_{e}=4181$, respectively. The plain line shows the numerical solution of the linear dispersion relation. Clearly, if there are not enough electron markers in the simulations, the linear response of the plasma is incorrect. For the simulation with only $N_{e}=233$ electron markers (diamonds in Figure 5) the agreement with theory is very good. To put this result in perspective, this corresponds to about $N_{e} / N \sim 0.5$ electron markers per cell! For 8 electron markers per cell (triangles), the linear growth rate agrees almost perfectly with the theoretical value. Therefore, the use of splitting scheme allows us to simulate drift-type modes with few electrons; this, of course, has a favorable impact on the development of global, particle-in-cell simulations in general toroidal geometry.

Figures 6 and 7 offer a different point of view of the splitting scheme. The parameters are $N_{i}=N_{e}=10946$, system length $L=8$, number of grid points $N=64$, driving parameter $\kappa=0.1$ and $\eta_{e}=\eta_{i}=0$. At $t=0$ a group of $M\left(\ll N_{e}\right)$ electron markers with $V_{\min }<V_{j}<V_{\max }$ 
is picked randomly from the initial distribution. As the simulation progresses, the position of these markers in velocity space is followed in time; time progresses in the counterclockwise direction for markers with positive initial velocities in Figures 6 and 7. The position in velocity space of marker $j$ at time step $t_{k}=k \Delta t$ is thus given by

$$
\begin{aligned}
V_{x j}^{(k)} & =V_{j}^{(k)} \cos \theta_{k} \\
V_{y j}^{(k)} & =V_{j}^{(k)} \sin \theta_{k}
\end{aligned}
$$

where $\theta_{k}=k \Delta t / T$ where $T$ is the total simulation time, and $V_{j}^{(k)}$ is obtained from the nonlinear simulations; for Figures 6 and $7, \Delta t=1.0$ and $T=4000$. In the absence of turbulence, all markers would describe perfect circles in velocity space. Figure 6 shows the trajectories in velocity space of 8 electrons markers chosen randomly in the interval $V_{\text {min }} / V_{\text {the }}=1.0<$ $V_{k}<V_{\text {max }} / V_{\text {the }}=1.2$; clearly these markers do not "see" the fastest growing mode, or even higher-order modes; in other words, they behave adiabatically. We have repeated the simulation with the same initial conditions in phase space and the same physical parameters for a set of 8 electron markers with $V_{\min } / V_{\text {the }}=0.1<V_{k}<V_{\max } / V_{\text {the }}=0.2$ (Figure 7 ). The resonant velocity, normalized to the electron thermal velocity, is $V_{r} / V_{t h e} \simeq \omega_{r} /\left(k_{\|} V_{t h e}\right)=0.144$. Clearly the slow electrons interact strongly with the waves in this case as their initial velocities are close to the resonant velocity $\omega_{r} / k_{\|}$. Figures 6 and 7 suggest that the diffusion in velocity space is strongly anisotropic. In order to quantity this assertion, one can extend the random walk argument usually used in configuration space to the velocity domain. One can define the diffusion coefficient in velocity space as

$$
D_{v}=\lim _{t \rightarrow \infty} \sum_{k=1}^{N_{e}} \frac{\left(\Delta V_{k}\right)^{2}}{2 N t}
$$

where $\Delta V_{k}(t)=V_{k}(t)-V_{k}(0)$ is the change in velocity of marker $k$ in time $t$. The velocity space diffusion coefficient as a function of time for a simulation with $N_{e}=N_{i}=10946$ markers is shown in Figure 8. For the parameters of Figure 8, the typical growth time associated with the fastest growing mode is $\tau_{\ell} \sim 1 / \gamma_{\ell} \approx 330$ (as before, $\gamma_{\ell}$ denotes the linear growth rate). Interestingly, $D_{v}$ saturates after approximately 14 growth times; the reason for this slow saturation is presumably due to the appearance of short wavelength modes that ultimately 'untrapped' trajectories such as that shown in Figure 6. As mentioned earlier, we expect the diffusion in velocity space to be strongly anisotropic. Consider a subset $M \ll N_{e}$ of electron markers with initial velocities in the interval $V_{\min }<V_{k}(0)<V_{\max }$ and define

$$
\widehat{D}_{v}\left(V_{\text {min }}, V_{\text {max }}\right) \equiv \lim _{t \rightarrow \infty} \sum_{k=1}^{M} \frac{\left(\Delta V_{k}\right)^{2}}{2 M t}
$$

Clearly, $\widehat{D}_{v}(-\infty,+\infty)=D_{v}$ when $M=N_{e}$. Table 1 shows the velocity space diffusion coefficient (23) (in units of $c_{s}^{2} \omega_{c i}$ ). The strong diffusion takes place near the resonant velocity, $V_{r}$, and $\widehat{D}_{v}$ drops dramatically away from $V_{r}$.

We complete our description of the splitting scheme by considering the overall energy conservation. Here again, it is convenient to compare the splitting scheme with the well-known $\delta f$ scheme [6] (Appendix). Figure 9 shows the kinetic energy (dashed line), potential (dotted line) and the total energy (plain line) obtained for a $\delta f$ simulation using $N_{i}=N_{e}=6765$ markers. A slow but steady rise in the total energy is observed. Of course, the energy conservation can be improved by increasing the number of markers per cell. Figure 10 shows the energy evolution 
obtained for the splitting scheme for the same parameters and the same initial conditions as those of Figure 9. The total energy does not display the steady rise observed in the $\delta f$ scheme and its overall magnitude is smaller than that of the $\delta f$ scheme case.

\section{Concluding Remarks}

The accurate treatment of electron dynamics in particle-in-cell simulations represents a major challenge since the fast electrons, $V_{t h e} / V_{t h i} \sim \sqrt{m_{i} / m_{e}} \gg 1$, imply a stringent condition on the time step. However, for low-frequency modes [2,3] that are relevant to the anomalous transport in tokamaks [1] and stellarators [4,12], the bulk of the electrons do not "see" the waves, that is, they respond adiabatically. Based on this observation, we have developed the splitting scheme, which segregates electrons into two subgroups (adiabatic electrons and nonadiabatic electrons). One consequence of the splitting algorithm is that nonlinear elliptic equations have to be solved; the solution of such nonlinear elliptic problems is best addressed using a multigrid solver.

It has been shown that the splitting scheme yields more accurate linear growth rates as those obtained with the conventional $\delta f$ scheme [6], and that simulations with few electron markers give satisfactory results. The favorable numerical trends of the splitting scheme are also reflected in the overall energy conservation.

This paper is part of an ongoing project for the self-consistent treatment of electron dynamics in large-scale, particle-in-cell simulations of tokamak and stellarator plasma microturbulence. Although a relatively simple model was presented in this paper, the main conclusion is that the multigrid particle-in-cell approach offers an attractive path for the simulation of plasma microturbulence, as the computation work scales roughly linearly with the number of markers and the number of grid points in configuration space, even for three-dimensional plasmas.

\section{Acknowledgments}

This research was supported by Contract No DE-AC02-76CH03073 and the Scientific Discovery through Advanced Computing (SciDAC) initiative (U.S. Department of Energy). 


\section{Appendix: Weight Equation for the Standard $\delta f$ Scheme}

In this Appendix, we derive the weight equation for the standard $\delta f$ scheme. We substitute the representation of

$$
F_{j}=F_{M j}+\delta f_{j}
$$

where $F_{M j}$ is the Maxwellian distribution, in the collisionless, Vlasov equation (1) and use

$$
\left(\frac{\partial}{\partial t}+v_{\|} \widehat{\mathbf{b}}_{0} \cdot \nabla\right) F_{M j}=0
$$

and $\boldsymbol{\nabla} F_{M j}=-\boldsymbol{\kappa}_{j} F_{M j}$ to show that (in gyrokinetic units)

$$
\frac{d \delta f_{j}}{d t}=\left(\mathbf{V}_{E} \cdot \boldsymbol{\kappa}_{j}+\sigma_{j} v_{\|} \nabla_{\|}\right) F_{M j}
$$

where $\sigma_{j} \equiv Z_{j} T_{e} / T_{j}$. The equation governing $W_{j} \equiv \delta f_{j} / F_{j}$ follows immediately from the above equation

$$
\frac{d W_{j}}{d t}=\left(1-W_{j}\right)\left[\left(\widehat{\mathbf{b}}_{0} \times \nabla \Phi\right) \cdot \boldsymbol{\kappa}_{j}-\sigma_{j} v_{\|} \nabla_{\|} \Phi\right]
$$




\section{References}

[1] P. C. Liewer, Nucl. Fusion 25, 543 (1985).

[2] W. Horton, Handbook of Plasma Physics (Vol.2) (Elsevier Science, Amsterdam, 1984).

[3] W. Horton, Rev. Mod. Phys. 71, 735 (1999).

[4] F. Wagner and U. Stroth, Plasma Phys. Contr. Fusion, 35, 1321 (1993).

[5] G. Hu and J.A. Krommes, Phys. Plasmas, 1(4), 863 (1994).

[6] R.E. Denton and M. Kotschenreuther, J. Comp. Phys. 119, 283 (1995).

[7] B.D. Fried and S.D. Conte, Plasma Dispersion Function (Academic Press, New York, 1961).

[8] F. De la Vallee Poussin, SIAM J. of Num. Analysis, 5(2), 340 (1968).

[9] A. Settari and K. Aziz, SIAM J. of Num. Analysis, 10(3), 506 (1973).

[10] W.S Press, S.A. Teukolsky, W.T. Vetterling and B.P. Flannery, Numerical Recipes in Fortran (Cambridge University Press, New York, 1992).

[11] S. Parker and W.W. Lee, Phys. Fluids, B5(1), 77 (1993).

[12] J.L.V. Lewandowski, Phys. of Plasmas, 7(8), 3360 (2000). 


\begin{tabular}{|l|l|l|}
\hline$V_{\min } / V_{\text {the }}$ & $V_{\max } / V_{\text {the }}$ & $\widehat{D}_{v}$ \\
\hline 0.0 & 0.2 & $4.9 \times 10^{-4}$ \\
\hline 0.2 & 0.4 & $1.5 \times 10^{-4}$ \\
\hline 0.4 & 0.6 & $2.7 \times 10^{-6}$ \\
\hline 0.6 & 0.8 & $1.1 \times 10^{-6}$ \\
\hline 0.8 & 1.0 & $5.5 \times 10^{-7}$ \\
\hline 1.0 & 1.2 & $3.5 \times 10^{-7}$ \\
\hline
\end{tabular}

Table 1: Velocity space diffusion coefficients in various velocity layers. 
Figure $1 L^{2}$ norm of the residual as a function of the number of $V$ cycles (iterations) for a grid with $N_{\theta}=256$ grid points. The number of relaxation sweeps on each level is $\nu=4$ and the Jacobi damping parameter is $\omega=0.6$. The initial profile is given by Eq.(20).

Figure 2 Linear growth rate for the standard $\delta f$ scheme (triangles) and for the splitting scheme (squares) as a function of $\kappa=\rho_{\mathrm{s}} / L_{n}$. The plain line is the numerical solution of the linear dispersion relation. The parameters are: $N_{e}=N_{i}=6765$, on a grid of length $L=8$ with 64 grid points; $\eta_{e}=\eta_{i}=0$ and $\theta=0.01$. Only the $N=1$ mode $\left(k_{\perp} \rho_{s} \simeq 0.78\right)$ is retained in the simulation. The initial configuration in phase space for the splitting scheme run and the $\delta f$ run are identical.

Figure 3 Time history of the real (plain line) and imaginary (dotted line) parts of the $n=1$ mode based on the splitting scheme (nonlinear run).

Figure 4 Saturation level of the Parker-Lee three-wave mode coupling theory [11] (plain line) compared with the measured values for a set of $N_{e}=N_{i}=6765$ markers (squares) and for a set of $N_{e}=N_{i}=4181$ markers (triangles) as a function of the drive, $\kappa$.

Figure 5 Linear growth rate as a function of $k_{\perp} \rho_{s}$ for a set of $N_{i}=10946$ ion markers; the computational grid has $N=512$ nodes. The number of electron markers is $N_{e}=144$ squares), $N_{e}=233$ (diamonds) and $N_{e}=4181$ (triangles); the plain line is the numerical solution (using Muller's algorithm [10]) of the linear dispersion relation, Eq.(12). Other parameters are $\kappa=0.15, \eta_{e}=0$ and $\eta_{i}=0$.

Figure 6 Trajectories of a set of 8 electron markers in velocity space (picked randomly from the initial distribution function) with initial velocities $1.0<V_{k}(0) / V_{\text {the }}<1.2$. The number of ion and electron markers is $N_{e}=N_{i}=10946$; other parameters are $\kappa=\rho_{s} / L_{n}=0.1$, $\eta_{e}=\eta_{i}=0, L=8$ and $N=64$.

Figure 7 Trajectories of a set of 8 electron markers in velocity space (picked randomly from the initial distribution function) with initial velocities $0.1<V_{k}(0) / V_{\text {the }}<0.2$. The number of ion and electron markers is $N_{e}=N_{i}=10946$; other parameters are $\kappa=\rho_{s} / L_{n}=0.1$, $\eta_{e}=\eta_{i}=0, L=8$ and $N=64$. The resonant velocity is $V_{r} / V_{\text {the }} \simeq \omega_{r} /\left(k_{\|} V_{\text {the }}\right)=0.144$.

Figure 8 Velocity space diffusion coefficient [Eq.(22)] for a nonlinear simulation with $N_{i}=$ $N_{e}=10946$ markers. The time step is $\Delta t=1.0$; the typical growth time of the fastest growing mode is $\tau_{\ell} \sim 1 / \gamma_{\ell} \simeq 330$.

Figure 9 Kinetic (dashed line), potential (dotted line) and total (plain line) for a nonlinear simulation with $N_{i}=N_{e}=6765$ markers using the $\delta f$ scheme.

Figure 10 Kinetic (dashed line), potential (dotted line) and total (plain line) for a nonlinear simulation with $N_{i}=N_{e}=6765$ markers using the splitting scheme, using the same initial conditions and physical parameters as in Figure 9. 


\section{FIG.1 Lewandowski}

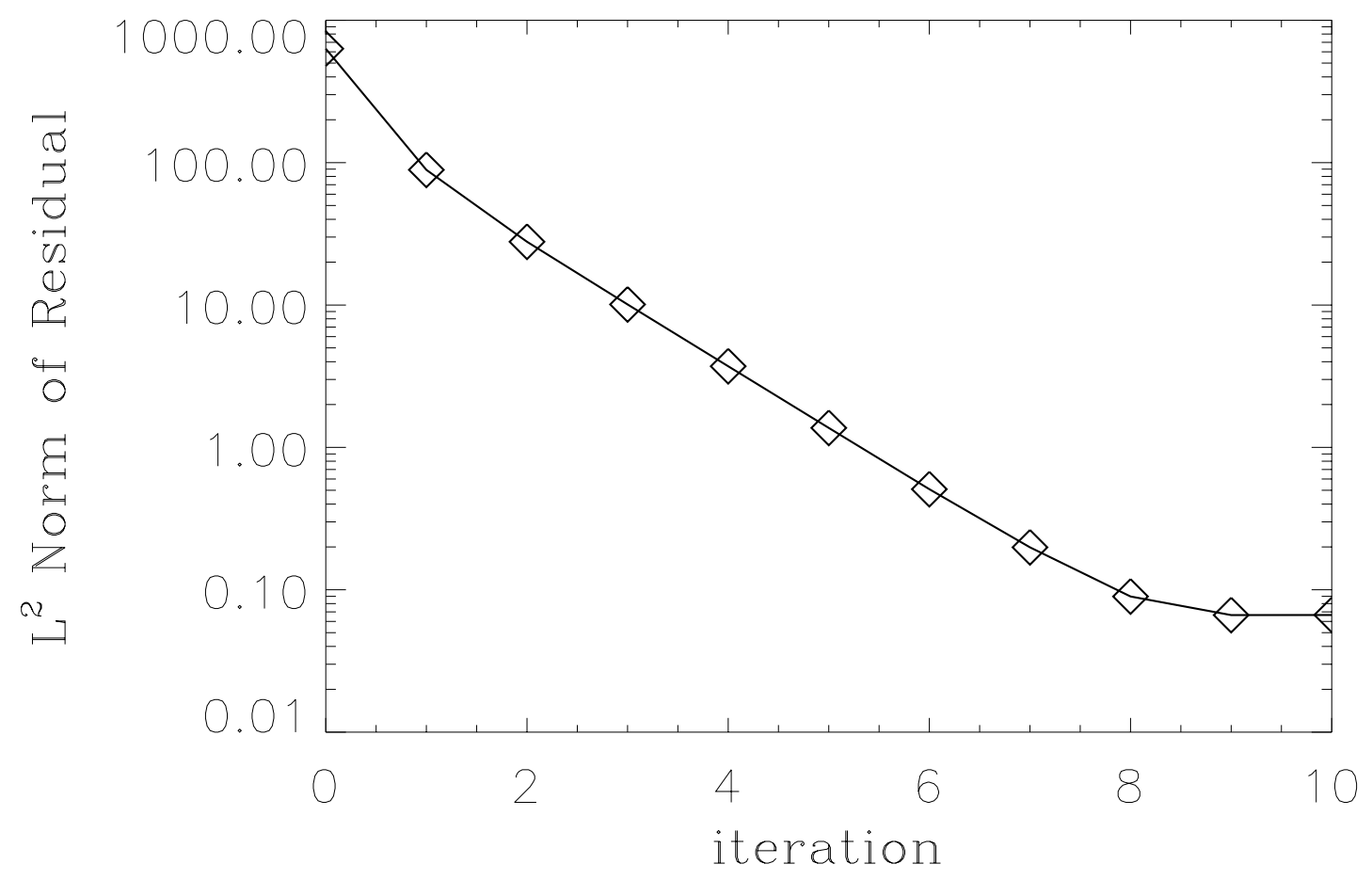




\section{FIG.2 Lewandowski}

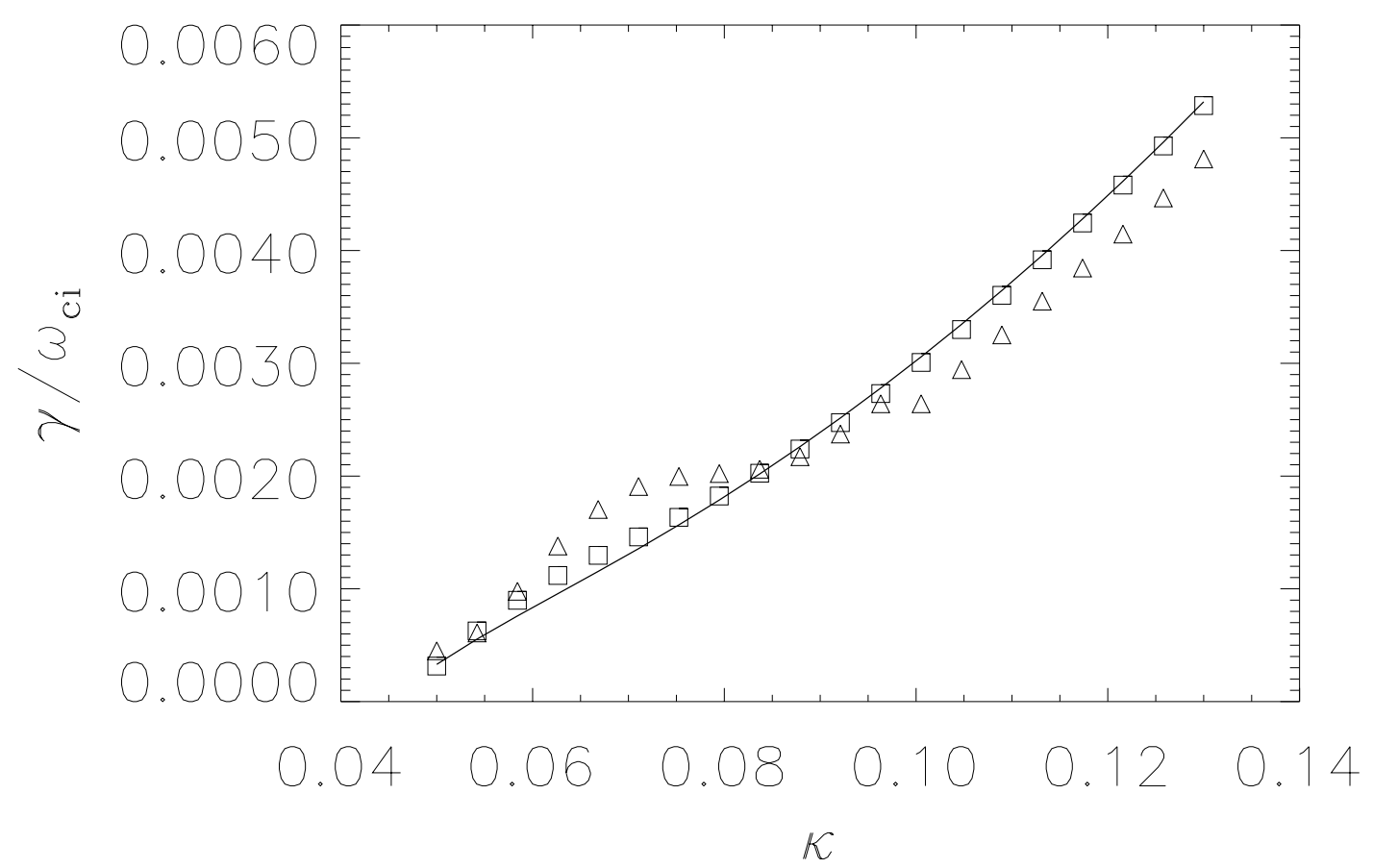


FIG.3 Lewandowski

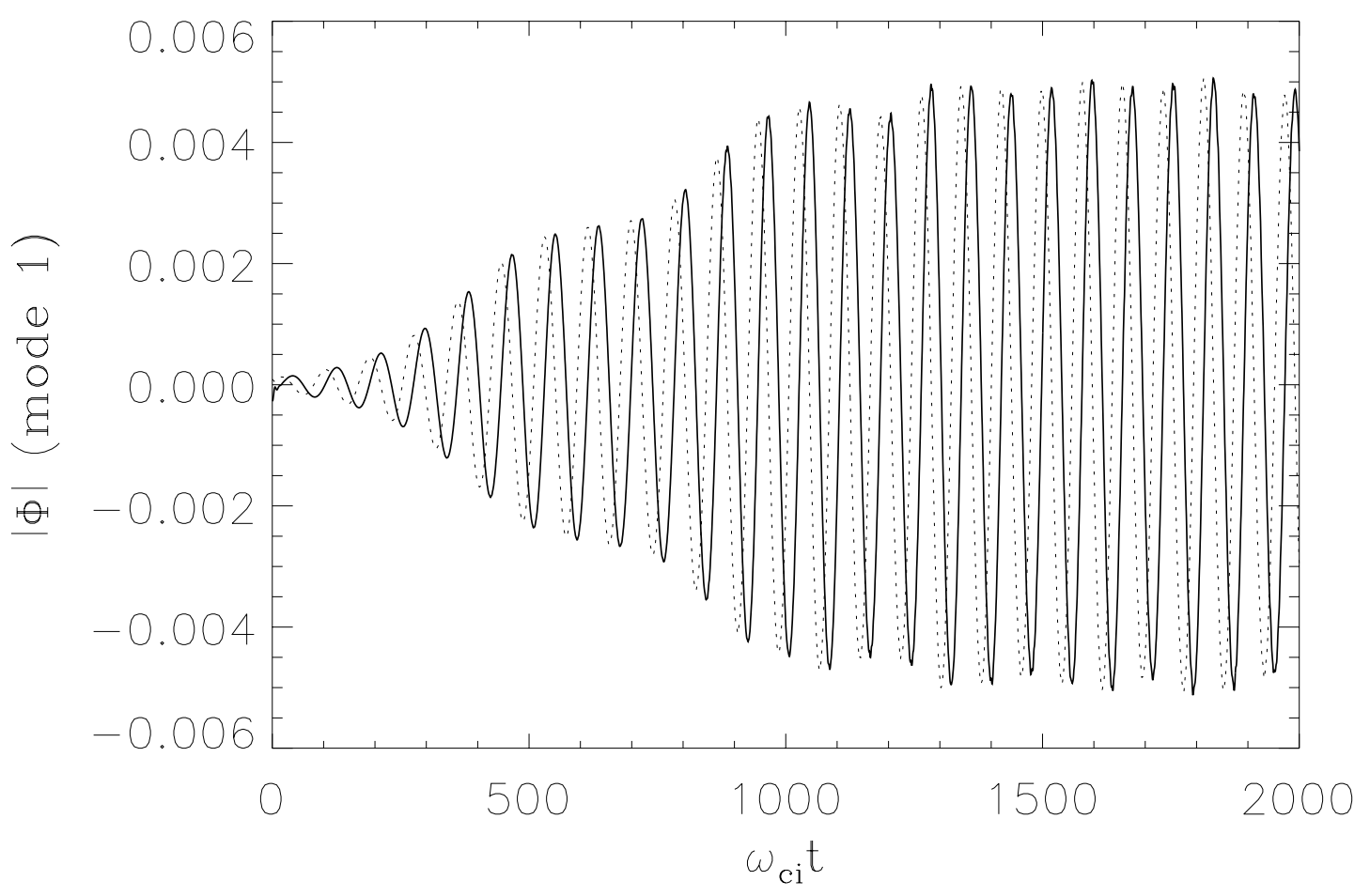




\section{FIG.4 Lewandowski}

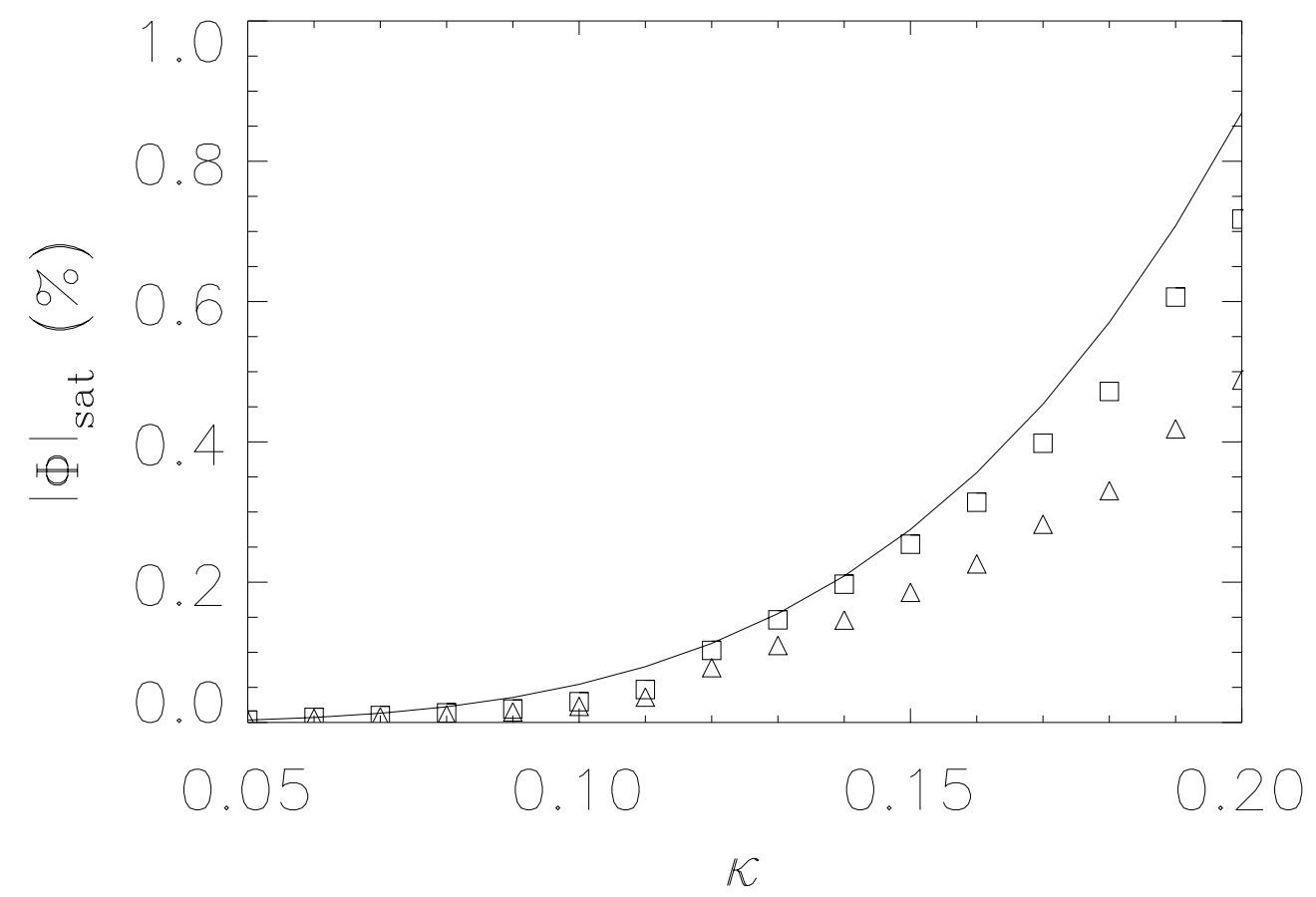




\section{FIG.5 Lewandowski}

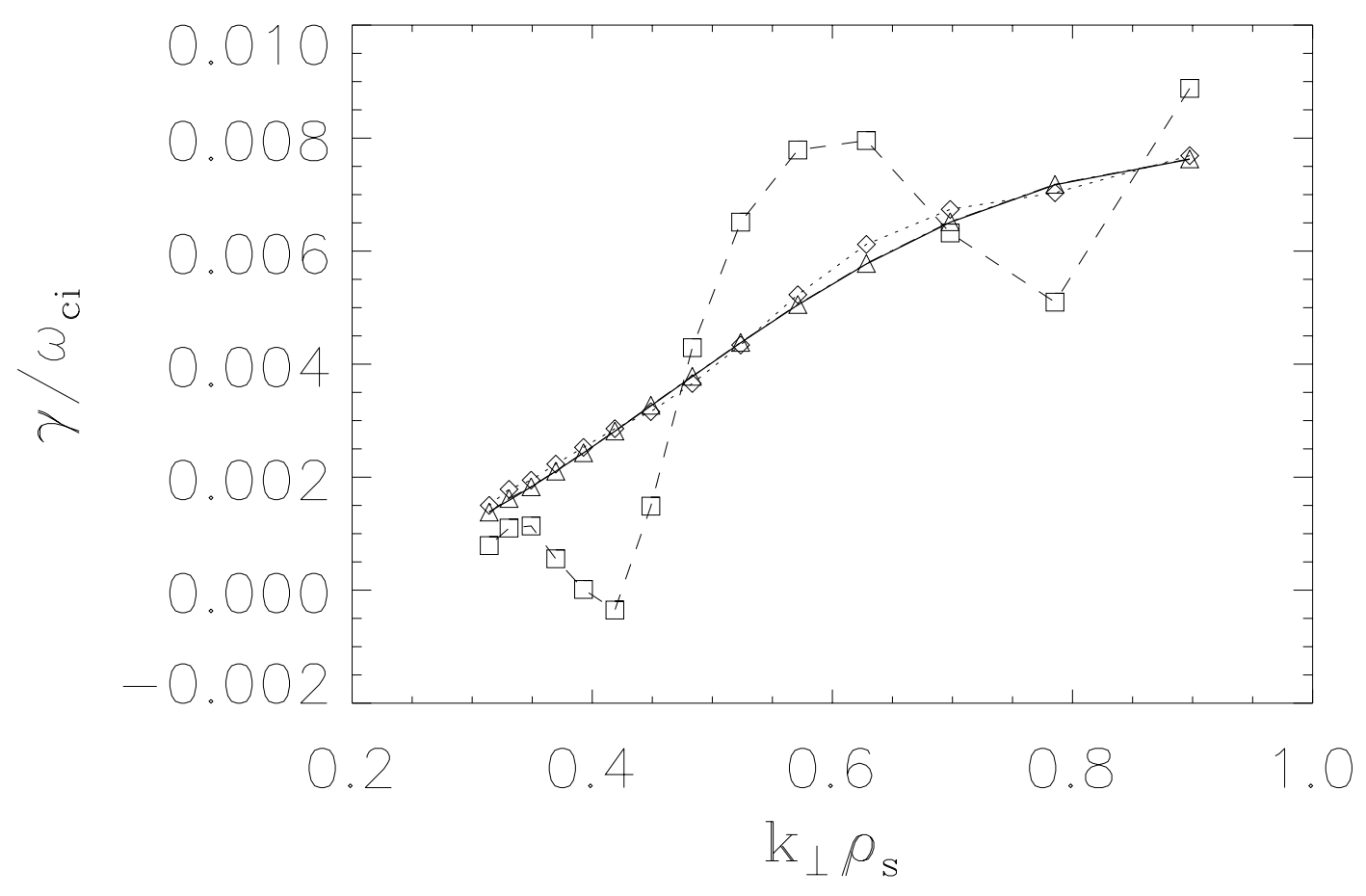




\section{FIG.6 Lewandowski}

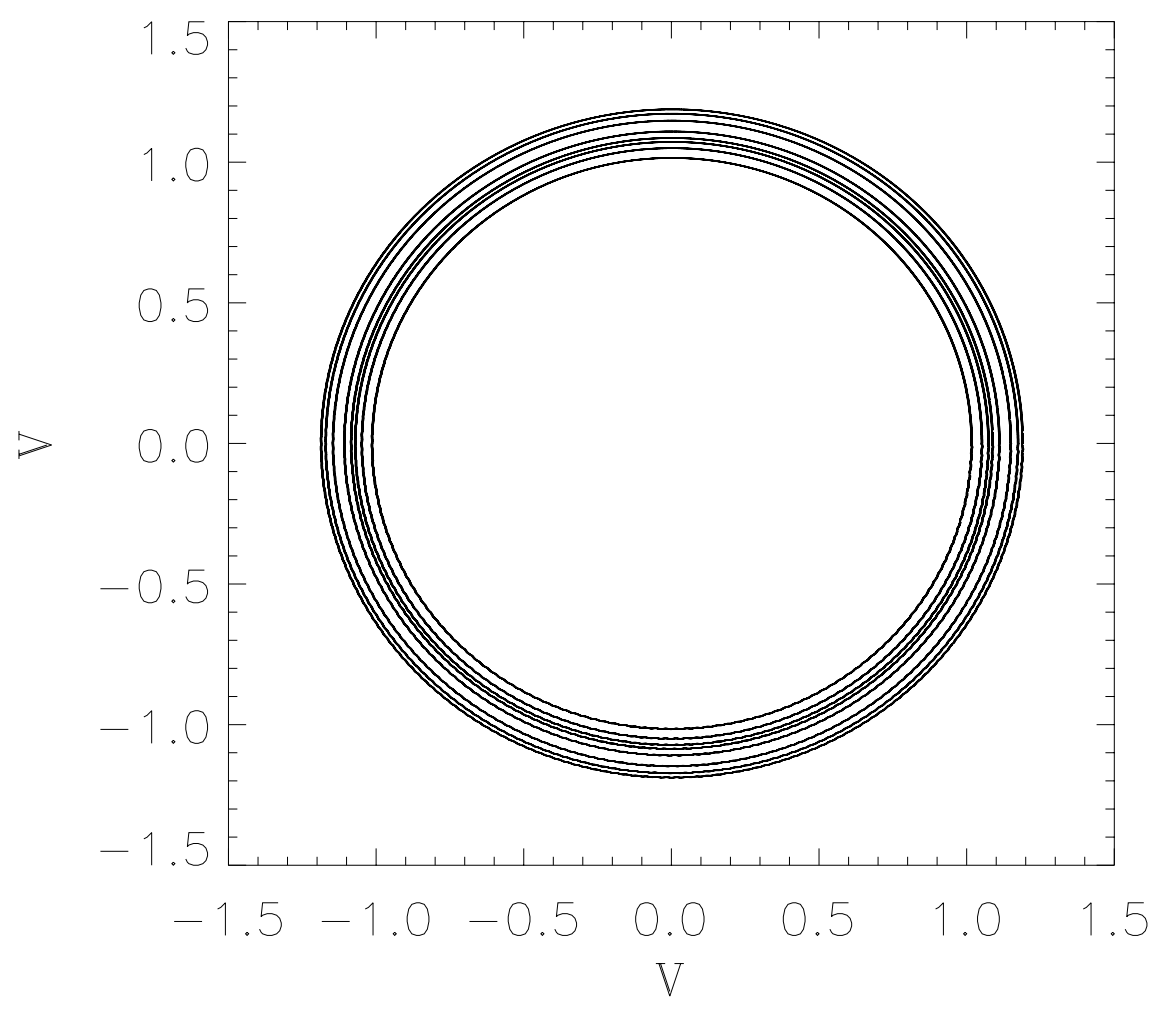




\section{FIG.7 Lewandowski}

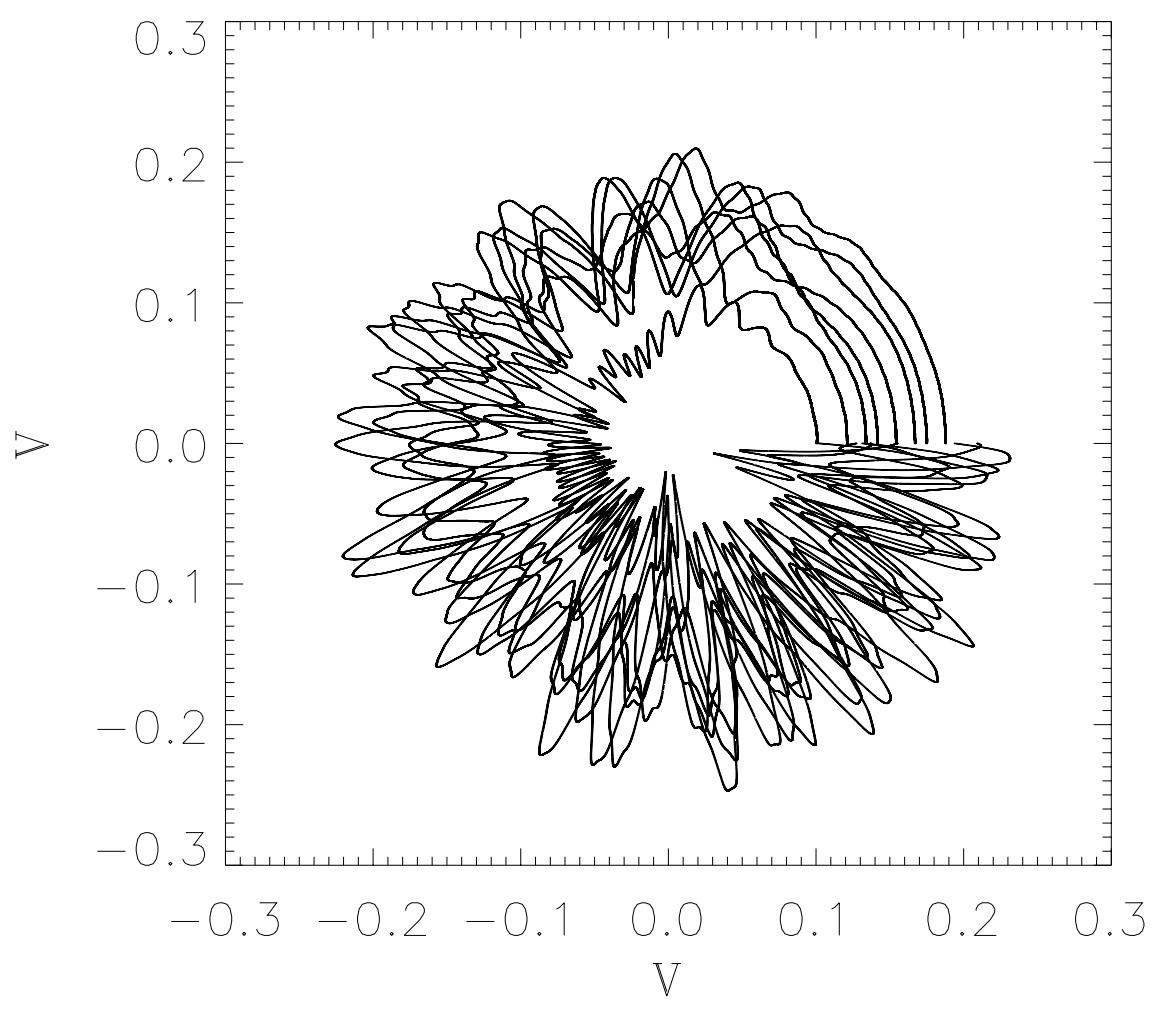


FIG.8 Lewandowski

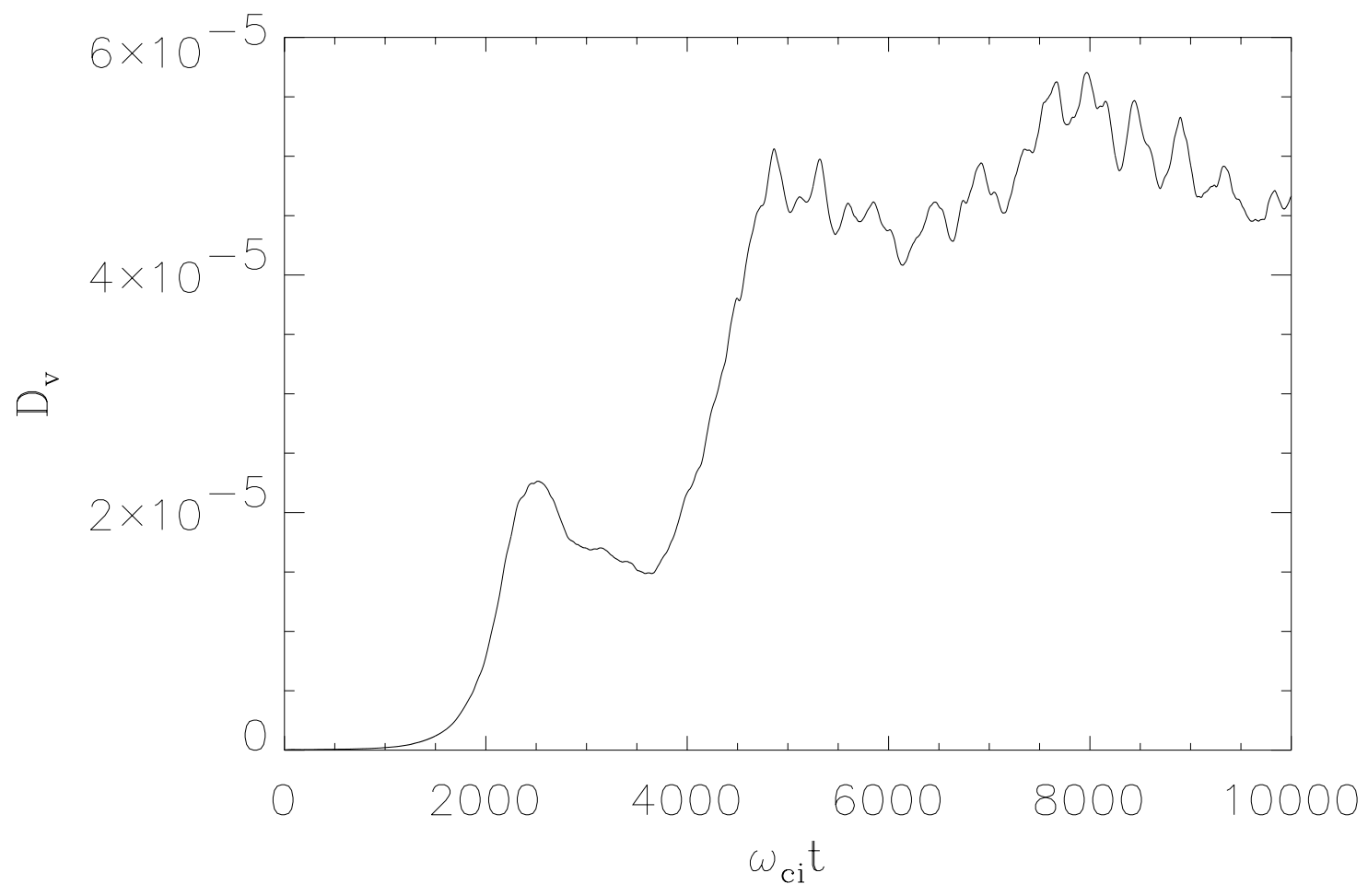




\section{FIG.9 Lewandowski}

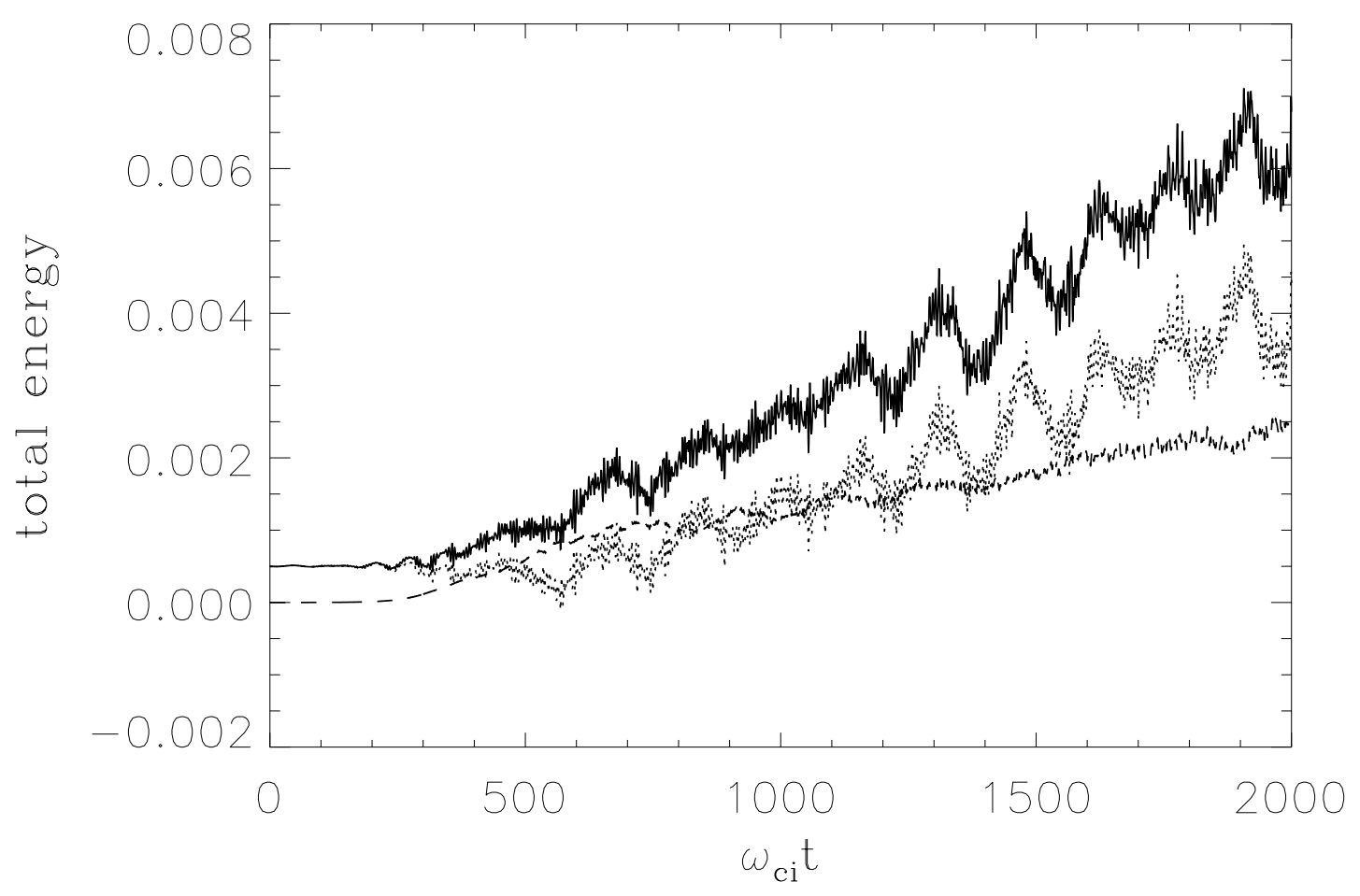




\section{FIG.10 Lewandowski}

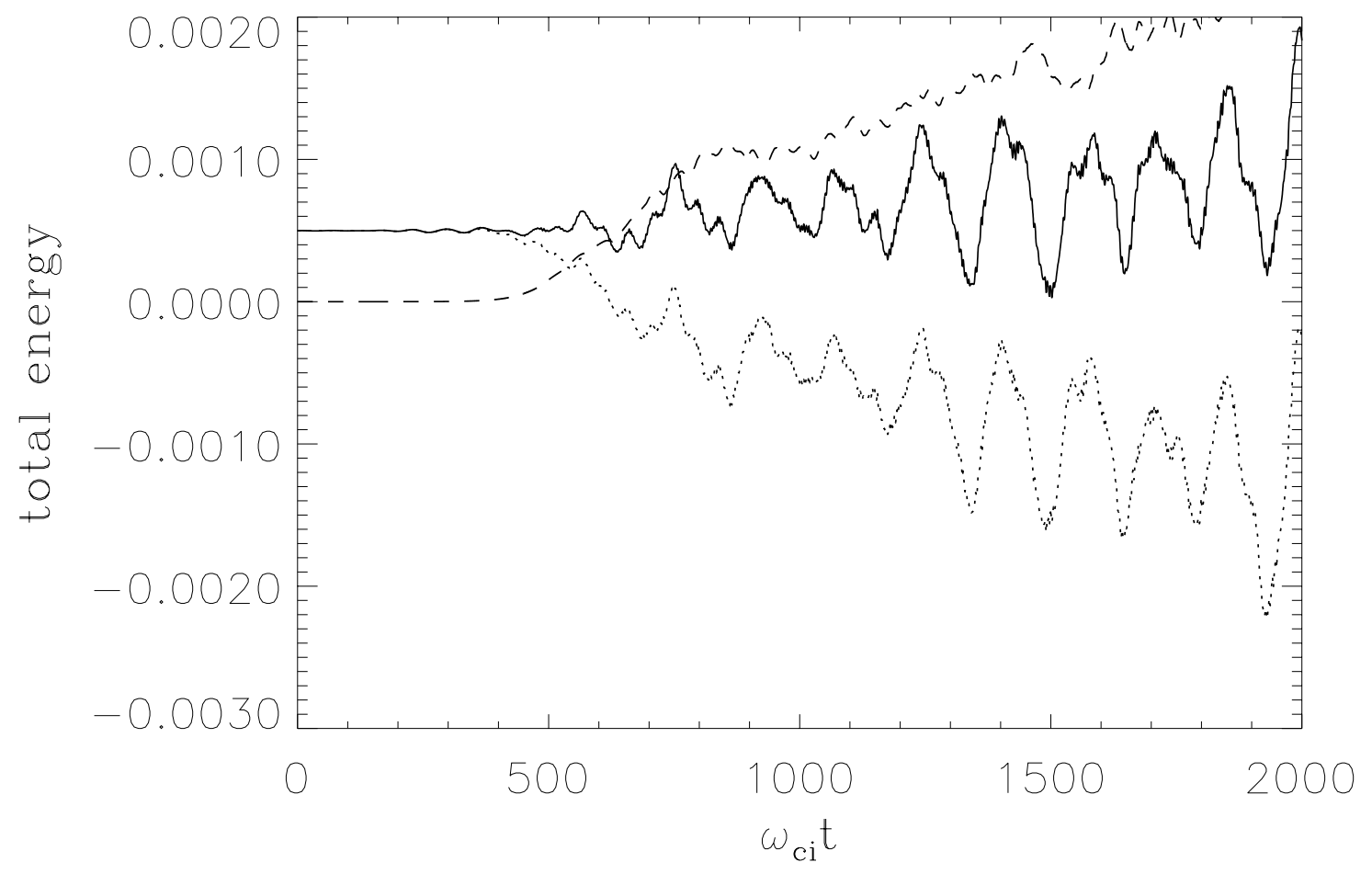




\section{External Distribution}

Plasma Research Laboratory, Australian National University, Australia

Professor I.R. Jones, Flinders University, Australia

Professor João Canalle, Instituto de Fisica DEQ/IF - UERJ, Brazil

Mr. Gerson O. Ludwig, Instituto Nacional de Pesquisas, Brazil

Dr. P.H. Sakanaka, Instituto Fisica, Brazil

The Librarian, Culham Laboratory, England

Mrs. S.A. Hutchinson, JET Library, England

Professor M.N. Bussac, Ecole Polytechnique, France

Librarian, Max-Planck-Institut für Plasmaphysik, Germany

Jolan Moldvai, Reports Library, Hungarian Academy of Sciences, Central Research Institute for Physics, Hungary

Dr. P. Kaw, Institute for Plasma Research, India

Ms. P.J. Pathak, Librarian, Institute for Plasma Research, India

Ms. Clelia De Palo, Associazione EURATOM-ENEA, Italy

Dr. G. Grosso, Instituto di Fisica del Plasma, Italy

Librarian, Naka Fusion Research Establishment, JAERI, Japan

Library, Laboratory for Complex Energy Processes, Institute for Advanced Study, Kyoto University, Japan

Research Information Center, National Institute for Fusion Science, Japan

Dr. O. Mitarai, Kyushu Tokai University, Japan

Dr. Jiangang Li, Institute of Plasma Physics, Chinese Academy of Sciences, People's Republic of China

Professor Yuping Huo, School of Physical Science and Technology, People's Republic of China

Library, Academia Sinica, Institute of Plasma Physics, People's Republic of China

Librarian, Institute of Physics, Chinese Academy of Sciences, People's Republic of China

Dr. S. Mirnov, TRINITI, Troitsk, Russian Federation, Russia

Dr. V.S. Strelkov, Kurchatov Institute, Russian Federation, Russia

Professor Peter Lukac, Katedra Fyziky Plazmy MFF UK, Mlynska dolina F-2, Komenskeho Univerzita, SK-842 15 Bratislava, Slovakia

Dr. G.S. Lee, Korea Basic Science Institute, South Korea

Institute for Plasma Research, University of Maryland, USA

Librarian, Fusion Energy Division, Oak Ridge National Laboratory, USA

Librarian, Institute of Fusion Studies, University of Texas, USA

Librarian, Magnetic Fusion Program, Lawrence Livermore National Laboratory, USA

Library, General Atomics, USA

Plasma Physics Group, Fusion Energy Research Program, University of California at San Diego, USA

Plasma Physics Library, Columbia University, USA

Alkesh Punjabi, Center for Fusion Research and Training, Hampton University, USA

Dr. W.M. Stacey, Fusion Research Center, Georgia Institute of Technology, USA

Dr. John Willis, U.S. Department of Energy, Office of Fusion Energy Sciences, USA

Mr. Paul H. Wright, Indianapolis, Indiana, USA 
The Princeton Plasma Physics Laboratory is operated by Princeton University under contract with the U.S. Department of Energy.

\author{
Information Services \\ Princeton Plasma Physics Laboratory \\ P.O. Box 451 \\ Princeton, NJ 08543
}

Phone: 609-243-2750

Fax: 609-243-2751

e-mail: pppl_info@pppl.gov

Internet Address: http://www.pppl.gov 